\title{
Long-term safety and efficacy of endovascular abdominal aortic aneurysm repair
}

\author{
This article was published in the following Dove Press journal: \\ Vascular Health and Risk Management \\ 2 April 2013 \\ Number of times this article has been viewed
}

\author{
Brandon W Propper \\ Christopher J Abularrage \\ Division of Vascular Surgery and \\ Endovascular Therapy, John Hopkins \\ Hospital, Baltimore, MD, USA
}

\begin{abstract}
Endovascular abdominal aortic aneurysm repair (EVAR) is a safe and efficacious treatment for both unruptured and ruptured abdominal aortic aneurysms. While perioperative mortality is lower with EVAR, long-term outcomes are similar between EVAR and open repair, including quality of life and cost-effectiveness. We review the long-term outcomes from the EUROSTAR registry, and DREAM, EVAR 1, and OVER trials.
\end{abstract}

Keywords: EVAR, endovascular, aneurysm, aortic, outcome, long-term

\section{Background}

Endovascular abdominal aortic aneurysm repair (EVAR) is currently the treatment of choice for repair of uncomplicated infrarenal abdominal aortic aneurysms (AAA). However, any current discussion must also recognize the great strides that have occurred in the past 22 years since Parodi and colleagues published their initial experience in 1991. ${ }^{1}$ In their initial report, they describe the customization of Dacron tube grafts affixed to balloon-expandable stents. Each patient's graft was custom made and individualized. While technology has provided an array of prefabricated devices, the preoperative measurements and planning are still individualized to each patient's anatomy. The purpose of this report is to discuss the long-term safety and efficacy of EVAR.

Initial endovascular therapy provides a less invasive treatment for many patients; however, almost $40 \%$ of patients are not suitable for endovascular repair. ${ }^{2}$ Industry has focused significant time and effort on modifying devices and accommodating difficult anatomic pathology. Initial devices required aneurysm neck lengths of at least $20 \mathrm{~mm}$ and neck angulation of less than $60^{\circ}$. Both of these constraints were overcome with more flexible grafts and the addition of proximal, suprarenal stents. Even newer devices have focused on lower-profile delivery systems to accommodate patients with smaller iliac vessels, and further modifications seek to provide entire aortic coverage. With each iteration, prior problems are addressed, but new complications are encountered and new challenges realized. While it is tempting to believe that new technology comes with fewer complications, long-term efficacy of endovascular repair must be evaluated as a whole.

\section{Timing of repair}

The primary goal of EVAR remains the prevention of rupture of AAA. The size of AAA strongly correlates with future risk of rupture, ${ }^{3}$ and as such, current recommendations utilize $5-5.5 \mathrm{~cm}$ as the threshold for repair. This size is generally felt to represent the point at which the risk of repair is less than the annual risk of rupture; however, 
the physician must consider individual risk. Prospective, randomized studies have shown no benefit to early open repair of aneurysms less than $5.5 \mathrm{~cm} .{ }^{4,5}$

As EVAR became more prevalent, two questions arose. First, since the mortality after EVAR was less than that of open repair, would it be appropriate to treat small AAA with endovascular stent grafts? Second, would nonoperative surveillance decrease the likelihood that a patient would be an EVAR candidate based on anatomic selection criteria? The PIVOTAL trial in the US randomized 728 patients to early EVAR or surveillance and found no difference in mortality or aneurysm-related mortality. ${ }^{6}$ Furthermore, the CAESAR trial in Europe had similar findings, with no clear advantage between early or delayed EVAR strategy. ${ }^{7}$ The probability of delayed repair was $59.7 \%$ at 36 months and $84.5 \%$ at 54 months. Within 3 years, $16.4 \%$ of patients in the surveillance arm were no longer EVAR candidates.

Knowledge of an aneurysm and its risk of rupture can be associated with a great deal of anxiety. Some have hypothesized that early EVAR for small AAA would overcome anxiety in such patients; however, this has not been borne out in randomized trials. The CAESAR trial found that the 36-item Short-Form Health Survey (SF-36) health-related quality of life (QoL) in patients allocated to early EVAR and surveillance was similar. ${ }^{8}$ Overall, a recent Cochrane review confirmed that there is no benefit to early treatment of small, asymptomatic aneurysms, and $5.5 \mathrm{~cm}$ should remain the threshold for both open repair and EVAR. ${ }^{9}$

\section{Long-term results}

The long-term results of EVAR have been evaluated by a handful of trials, but four trials form the backbone of current endovascular therapy (Tables 1 and 2). The following is a synopsis of those four trials and the major findings.

\section{EUROSTAR}

The European Collaborators on Stent-Graft Techniques for Abdominal Aortic Aneurysm Repair (EUROSTAR) ${ }^{10}$ series is comprised of a multicenter registry from 62 European centers of EVAR with first-generation devices. Data from these centers was collected for over 3 years, starting in 1996 and ending in 1999. Almost $60 \%$ of the patients in this series were considered medically high-risk, established by an American Society of Anesthesiologists classification of III or higher. Conversion to open operation was reported in $7.1 \%$ of the cohort, which by current standards is exceptionally high; however, postprocedural aneurysm rupture occurred in $2.4 \%$, which is closer to current standards. Additionally, aneurysm-related death, defined as death within 30 days or related to any secondary procedure, was 3\%. Total follow-up time for the study was 8 years, with mean follow-up of 38 months. Secondary procedures were common, and occurred more frequently in those with aneurysms larger than $5.5 \mathrm{~cm}$. When $5.5 \mathrm{~cm}$ was used to divide the group, those with AAA $>5.5 \mathrm{~cm}$ had higher operative mortality $(4.4 \%$ vs $1.4 \%, P=0.002$ ).

Generally speaking, the EUROSTAR series reflects an early look at the first generation of infrarenal devices, and the inherent complications that occur with widespread use of new technology. The overall data reflect patients with aneurysms ranging from $4 \mathrm{~cm}$ and greater; however, current practice does not favor repair for those patients with AAA less than $5 \mathrm{~cm}$. Moreover, when reviewing the EUROSTAR registry, one might focus on the subgroup with AAA larger than $5.5 \mathrm{~cm}$, as this would better represent the current patients eligible for EVAR. While the main conclusion of the EUROSTAR group was the need for long-term surveillance due to the high incidence of complications requiring secondary procedures, especially in those treated with larger AAA $(>5.5 \mathrm{~cm})$, this can be explained by the selection criteria. A careful examination of the data shows that the $>5.5 \mathrm{~cm}$ subgroup had a greater number of preoperative comorbidites, shorter, wider, and more angulated aortic necks, and iliac access that was more tortuous. Based on current practice, it is not surprising that the $>5.5 \mathrm{~cm}$ subgroup, mostly those who would currently fall outside of the device instructions for use, had inferior results. ${ }^{11}$

Table I Trials comparing endovascular abdominal aortic aneurysm repair and open abdominal aortic aneurysm repair

\begin{tabular}{llllll}
\hline & Study design & Location & Enrollment dates & Patients & Maximum follow-up \\
\hline EUROSTAR & Registry & Europe & $1996-1999$ & 1190 & 8 years \\
DREAM & RCT & Netherlands and Belgium & $2000-2003$ & 351 & 6 years \\
EVAR I & RCT & United Kingdom & $1999-2004$ & 1252 & 10 years \\
OVER & RCT & US VAMC & $2002-2008$ & 881 & 9 years \\
\hline
\end{tabular}

Abbreviations: RCT, randomized controlled trial; US VAMC, United States Veterans Affairs Medical Centers. DREAM, Dutch Randomised Endovascular Aneurysm Management; EUROSTAR, European Collaborators on Stent-Graft Techniques for Abdominal Aortic Aneurysm Repair; EVAR, Endovascular abdominal aortic aneurysm repair; OVER, Open Surgery Versus Endovascular Repair of Abdominal Aortic Aneurysm. 
Table 2 Survival in randomized controlled trials

\begin{tabular}{rlll}
\hline Trial & $\begin{array}{l}\text { Early } \\
\text { survival }\end{array}$ & $\begin{array}{l}\text { Long-term } \\
\text { survival* }\end{array}$ & $\begin{array}{l}\text { Loss of EVAR } \\
\text { survival advantage }\end{array}$ \\
\hline $\begin{array}{r}\text { DREAM } \\
\text { Open }\end{array}$ & $4.6 \%$ & $69.9 \%$ & 2 years \\
EVAR & $1.2 \%^{\dagger}$ & $68.9 \%$ & \\
EVAR I & & & 6 months \\
Open & $4.7 \%$ & $57.8 \%$ & \\
EVAR & $1.7 \%^{\dagger}$ & $58.5 \%$ & 3 years \\
OVER & & & \\
Open & $3 . \%^{\dagger}$ & $66.6 \%$ & \\
EVAR & $0.5 \%^{\dagger}$ & $67.1 \%$ &
\end{tabular}

Notes: ${ }^{\text {DDREAM }}=6$ years, EVAR I $=8$ years, OVER $=9$ years; ${ }^{\dagger} P<0.05$ Abbreviation: DREAM, Dutch Randomised Endovascular Aneurysm Management; EVAR, endovascular abdominal aortic aneurysm repair; OVER, Open Surgery Versus Endovascular Repair of Abdominal Aortic Aneurysm.

\section{DREAM}

The Dutch Randomised Endovascular Aneurysm Management (DREAM) ${ }^{12-14}$ trial was a prospective randomized study that started in 2000 and enrolled 351 patients, ending in 2003. The study was conducted in 24 centers in the Netherlands and four hospitals in Belgium. Unlike EUROSTAR, the patients in DREAM all had AAA measuring at least $5 \mathrm{~cm}$ in diameter, with a mean diameter of $6 \mathrm{~cm}$. Additionally, patients in DREAM were randomized into either open repair or EVAR. Comorbid conditions were similar between both the open and endovascular groups. Over $90 \%$ of the patients enrolled were male, with an average age of 70 years. All endovascular repairs were conducted with devices that were approved by the US FDA or had an investigational device exemption. When compared to open repair, EVAR had shorter procedure times, less blood loss, fewer intensive care unit (ICU) days, and shorter lengths of stay. Additionally, transfusion requirement was less in the EVAR group. Operative mortality, defined as within 30 days or within the same hospital stay, was $4.6 \%$ for the open group and $1.2 \%$ for the EVAR group.

While systemic complications were higher in the open group, the need for secondary intervention was higher in the EVAR group. This was accounted for by endograft-related complications, including endoleak and graft migration.

Follow up at 2 years revealed an aneurysm-related mortality of $5.7 \%$ for the open group and $2.1 \%$ for the EVAR group $(P=0.05)$. Cumulative survival at 2 years was $89.6 \%$ for the open group and $89.7 \%$ for the EVAR group (not significant). In the publication of the 6-year follow-up, there was a significant difference in the freedom from secondary intervention between the open and EVAR groups $(81.9 \%$ vs $70.4 \%, P=0.03$ ). The cumulative 6 -year survival in the two groups remained similar, at $69.9 \%$ for the open group and $68.9 \%$ for the EVAR group (not significant).
The primary conclusion of DREAM was that EVAR had favorable early postoperative outcomes, with less early mortality and systemic complications. When compared with EUROSTAR, DREAM gives a more current assessment of EVAR. The devices used are largely similar to current EVAR devices with minor modifications. Furthermore, the DREAM investigators concluded that the short-term survival benefit was at the expense of an increase in the secondary complication rate in the EVAR group. Although this resulted in an increase in secondary interventions, there were no differences in long-term survival.

\section{EVAR I}

The EVAR $1^{15,16}$ trial originated from 37 centers throughout the UK. It was a prospective randomized trial that enrolled a total of 1252 patients between 1999 and 2004. Follow-up included a median time of 6 years (range 5-10 years). Patients were randomly assigned to either open or endovascular repair. Baseline comorbidities and characteristics were similar between both groups. Mean age was 74 years, with over $90 \%$ of the patients being men.

Compared to DREAM, the average aortic diameter was greater, at $6.4 \mathrm{vs} 6.0 \mathrm{~cm}$. Similar to DREAM, the majority of endografts used for repair were second- and third-generation devices with prior FDA approval. Thirty-day mortality for the open group was $4.7 \%$ compared with $1.7 \%$ in the EVAR group.

Total mortality and aneurysm-related mortality was calculated using Cox regression analysis. Total mortality for the open group was 7.7 deaths per 100 patient-years, with 7.5 deaths per 100 patient-years in the EVAR group. Total mortality was similar, and the authors attributed this equilibration to late graft rupture in the EVAR group. Aneurysm-related mortality was 1.0 deaths per 100 patient-years in the EVAR group compared to 1.2 in the open group (not significant).

Reintervention was almost five times more common in the EVAR group $(5.1 \%$ vs $1.7 \%, P<0.001)$. Interestingly, the majority of these reinterventions happened within 6 months following the index procedure.

The general conclusions of the EVAR 1 trial stressed the advantage of EVAR within the first 30 days, but further delineated similar findings to DREAM in terms of long-term mortality data. In the late postoperative period, the EVAR group had late ruptures that were seen far less frequently than in the open group. Critics of the EVAR 1 trial have noted that hernia repair is the most common cause of secondary intervention in open surgery (noted in both DREAM and OVER), and without this data, secondary procedure analysis is inaccurate. 


\section{OVER}

The Standard Open Surgery Versus Endovascular Repair of Abdominal Aortic Aneurysm (OVER) $)^{17,18}$ trial is the only trial of this group conducted inside the US. The OVER trial randomized patients at 42 Veterans Affairs hospitals to EVAR versus open repair. Patients had to be suitable for both types of repair, including being medically fit for open repair and meeting current instructions for use for endovascular device selection. A total of 881 patients were enrolled, and enrollment criteria included AAA $>5.0 \mathrm{~cm}, 4.5 \mathrm{~cm}$ with rapid expansion, or saccular aneurysm. Breakdown by size was as follows: $<5 \mathrm{~cm}, 5 \%$ of the cohort; $<5.5 \mathrm{~cm}, 43 \%$ of the cohort; and $>5.5 \mathrm{~cm}, 57 \%$ of the cohort. Baseline characteristics of the two groups were similar. Much like EVAR 1 and DREAM, the patients in the OVER trial were almost all male (99\%) and $87 \%$ white. Average age was $70 \pm 8$ years.

The total procedure time was lower in the EVAR group (2.9 vs 3.7 hours). Hospital length of stay, ICU stay, and ventilation times were all lower in the EVAR group. Average blood loss in the open group was $1 \mathrm{~L}$ compared to $200 \mathrm{~mL}$ in the EVAR group, which translated into a blood-transfusion difference of 0 units in the EVAR group vs 3 units in the open group.

Thirty-day operative mortality and hospital mortality was less in the EVAR group $(0.5 \%$ vs $3 \%, P=0.004)$. This statistical difference was not sustained for all-cause mortality. The EVAR group had 7\% all-cause mortality, versus $9.8 \%$ in the open group. The authors further stratified risk of death based on age greater than or less than 70 years, and aneurysm size greater than or less than $5.5 \mathrm{~cm}$. Younger patients tended to do better with EVAR, as did those with smaller aneurysms.

Unlike EVAR 1 and DREAM, secondary interventions were not different between the two groups (EVAR 13.7\% vs open $12.5 \%$ ). Of the EVAR group, there were 134 endoleaks documented in 110 patients. The majority of secondary procedures in the EVAR group were done via an endovascular approach. In the initial report, there was no difference in secondary procedures between open and endovascular repair, ${ }^{17}$ and later publications with longer follow-up of the same cohort continued to show no difference in secondary procedures. ${ }^{18}$ Erectile dysfunction and health-related QoL were not different at 2-year follow up between the two groups.

The OVER trialists concluded that both EVAR and open repair resulted in similar long-term survival. The perioperative mortality was lower in the EVAR group and sustained for several years, but late rupture remained a concern for the EVAR group. The more recent publication from the OVER group also identified a lower mortality for EVAR in patients under 70 years old. While the EVAR 1 trial showed no age-related difference, this is consistent with the EVAR 1 findings that "fit" patients had superior results and older patients had more complications. Interestingly, the OVER trialists also noted that patients over 70 years old who received open surgery tended to have lower mortality. This finding was unexpected, and the authors believe this tendency occurred because the majority of late EVAR ruptures occurred in patients over 70 years of age.

The OVER trial and later publications from this group represent the most recent data comparing EVAR and open surgery. This study extends the previous literature from DREAM and EVAR 1. EVAR 1 reviews have contended that without late rupture in the EVAR group, EVAR would have shown improved long-term survival, but the OVER trial disputes this finding. In the OVER trial, there were only three fatal late ruptures, yet long-term survival remained similar.

When viewed together, a total of 2443 patients were included, and all three prospective randomized trials failed to show any long-term survival advantage to EVAR; however, all showed decreased perioperative and 30-day mortality with EVAR.

\section{Quality of life}

Many have hypothesized that the minimally invasive nature of EVAR would translate into an improved postoperative QoL; however, this has not been borne out in these randomized trials.

The DREAM trial examined QoL at 1 year postoperatively using the EuroQol 5 Dimensions (EQ-5D) questionnaire and the SF-36. ${ }^{19}$ Both the EVAR and open groups had an initial decrease in QoL after surgery; this rebounded more quickly in the EVAR group. However, the open group reported a significantly higher score on the EQ-5D than the EVAR group from 6 months onward. There was also a marginal, nonsignificant benefit of open repair per quality adjusted life-year (QALY) (0.73 QALY) compared to EVAR (0.72 QALY).

The OVER trial, similarly, examined QoL using the EQ-5D and the SF-36. ${ }^{17,18}$ They found no differences in QoL at 6 months or 1,2, and 8 years postoperatively. Furthermore there were no differences in the 2-year QALY between the two groups (1.462 EVAR vs 1.461 open).

\section{Cost}

EVAR began as an alternative to open surgery in those patients considered high-risk. However, the trials discussed above have shown that it is a viable alternative for all patients. With its widespread use and high device cost, the question becomes whether or not EVAR is cost-effective in the long-term. 
The EVAR 1 trial found no difference in the mean cost of the primary aneurysm repair between the two groups (£13,019 EVAR vs $£ 11,842$ open). ${ }^{16}$ Not surprisingly, the mean cost of aneurysm-related readmissions was significantly higher in the EVAR group ( $£ 2283$ vs $£ 442$ ). Over the 8-year follow-up period, the total average cost of aneurysm-related procedures in the EVAR group was $£ 3019$ more than in the open group. The primary admission and readmissions for graft-related reinterventions contributed almost equally to the cost difference. As noted earlier, however, EVAR 1 did not include hernia repair, which has the potential to add significant cost to the open group.

The DREAM trial compared costs of 170 patients from the EVAR group and 170 patients from the open group. ${ }^{20}$ They found that EVAR was associated with a $€ 4293$ increase in direct costs compared to open repair. Not surprisingly, the increase in costs was related to both the cost of the device and the cost of postoperative surveillance, which was only partially compensated by the decrease in length of ICU and hospital stay. In terms of overall cost-effectiveness, open repair resulted in an improved overall cost-effectiveness ratio of $€ 76,100$ and $€ 171,500$ for costs per life-year gained.

The mean cost of hospital admission during the OVER trial was less in the EVAR group compared to the open group, despite a higher device cost (total cost $\$ 37,068$ vs $\$ 42,970) .{ }^{21}$ By 2 years, however, the decreased costs in the EVAR group $(-\$ 5,019)$ were no longer significant. Due to the lower costs and greater life-years in the EVAR group, the OVER trial did not calculate the cost per life-year saved. Based on bootstrap analysis, the probability of EVAR being less costly and more effective was $70.9 \%$ for life-years and $51.4 \%$ for QALYs. It should be noted that the OVER trial reported all health-care costs, rather than those strictly related to AAA repair.

\section{EVAR in the unfit}

Initial thoughts behind EVAR assumed that it would be more advantageous in the elderly and in those patients unfit for open repair. This debate continues, and practically speaking there are many patients who are currently offered EVAR but are not offered open repair. The EVAR 2 trial attempted to answer this question by comparing EVAR to observation with medical therapy in those unfit for repair. ${ }^{22}$ A total of 197 patients were assigned to EVAR, while 207 patients were observed without repair. Of those planned for observation, over 25\% ultimately underwent EVAR, the majority of surgeries occurring because of patient preference. Post hoc analysis of crossover patients indicated these patients to be more fit than those who did not cross over. As such, the authors stated that the crossover resulted in a loss of equipoise. With that in mind, the 30-day operative mortality for EVAR was $7.3 \%$, which is considerably higher than the previously mentioned EVAR trials. In addition, aneurysmrelated death was lower in the EVAR group; however, total mortality was similar between the two groups. Unfit patients undergoing EVAR had an almost 50\% rate of graft-related complications, and $27 \%$ required reintervention. Total cost was higher in the group undergoing EVAR.

The results of this trial showed that EVAR can be performed in those unfit for open repair, but the high complication rate and reintervention rate should be considered. Additionally, although aneurysm-related death was lower in the EVAR group, this did not translate into improved survival for the "unfit" patient.

\section{EVAR in the elderly}

As mentioned, patients over 80 years of age were thought to represent a cohort in whom EVAR would show substantial benefit. No prospective trials have been conducted to evaluate this, but subgroup analysis of larger cohorts has shed light on this particular issue. ${ }^{23}$ The Endurant Stent Graft Natural Selection Global Postmarket Registry (ENGAGE) registry consists of patients enrolled in Europe, Asia, and South America. A total of 274 patients were identified as over 80 years old and compared to those 80 years old or less. Only one particular device was used for all procedures. Technical success was achieved in over $99 \%$ of cases. The older group had higher American Society of Anesthesiologists classification, larger aneurysms, and more tortuous anatomy. All-cause mortality was similar between the two groups, and there was no difference in rupture or secondary procedures at 30 days. Overall, the older group had longer hospital stay and surgical operating times. The researchers concluded that EVAR can be performed safely in octogenarians.

Although initial data from the ENGAGE registry looks satisfying, it is important to note that these data represent 30-day follow up. Long-term comparisons were not made, and no comparison of long-term mortality was conducted. Furthermore, ENGAGE was a voluntary registry, rather than a randomized trial. While age often portends more medical comorbidities, it is not a surrogate for level of fitness. Age should be included when considering patients for EVAR, but overall fitness level (EVAR 2) is more important in determining the likelihood of success.

\section{Ruptured AAA}

Ruptured AAA (rAAA) continues to be a difficult challenge. Multiple reports have been published, and EVAR for rAAA 
has quickly become accepted as a viable treatment. A recent study from Albany reported on a single-center experience comparing EVAR and open surgery for rAAA. ${ }^{24}$ Over a 9-year period, EVAR was used in 120 patients and open surgery in 163 patients. In this group, EVAR for rAAA had a 30 -day mortality rate of $24.2 \%$ versus $44.2 \%$ for open surgery $(P<0.005)$. In addition, cumulative 5-year survival was better in the EVAR group ( 37 vs $26 \%, P<0.005$ ). The authors note important paradigm shifts in the treatment of rAAA. For example, early correction of coagulopathy, minimizing heparin use, and vigilance in identifying abdominal compartment syndrome has helped to decrease the early mortality. It should be noted that this group has extensive experience with EVAR for rAAA, and results may not be translatable to small or rural hospitals.

Another study examined all rAAA at two large European institutions. ${ }^{25}$ They found a statistical decrease in mortality of patients undergoing EVAR for rAAA compared to open surgery. Interestingly, there were differences in the analysis based on whether patients undergoing EVAR needed abdominal decompression for compartment syndrome. For patients not needing abdominal decompression, open surgery was associated with an increased mortality compared to EVAR (adjusted odds ratio =5.6). However, in patients with abdominal decompression by laparotomy, there was no difference in mortality (adjusted odds ratio $=1.1$ ). These results outline the wide spectrum of presentation seen in rAAA. Patients with symptomatic aneurysms or contained ruptures seem to do better with EVAR than those with frank rupture.

To improve upon the statistical quality of single-center studies, meta-analyses have been performed to compare EVAR and open surgery for rAAA. One such study performed a pooled analysis of eleven studies, comprising 42,888 patients. $^{26}$ They showed that EVAR was associated with significantly lower mortality than open surgery. When the population-based data were examined separately, this difference remained. However, when the analysis included only hospital-based data, EVAR had a trend toward lower mortality in the hospital-based subgroup that did not reach statistical significance.

While EVAR may be feasible for repair of rAAA, randomizing patients to EVAR or open surgery for rAAA has been fraught with difficulties. In one trial out of the UK, Hinchliffe and colleagues were only able to enroll 32 of 103 patients who were admitted with suspected rAAA. ${ }^{27}$ Overall, there were no differences in 30-day mortality and complications between EVAR and open surgery. The Amsterdam Acute Aneurysm trial randomized 83 of 105 patients with rAAA.$^{28}$ However, 45 (54\%) of the patients were not eligible for EVAR due to unsuitable aortic necks or iliac access. Further randomized studies, such as the IMPROVE trial, ${ }^{29}$ comparing EVAR and open surgery for rAAA are being performed to adequately answer the question of which technique provides the best long-term results.

\section{Conclusion}

EVAR is now considered the standard repair for uncomplicated infrarenal AAA. Despite rapid changes in technology, long-term survival remains similar between EVAR and open repair. Perioperative mortality is lower with EVAR, although secondary interventions are common. Long-term outcomes are similar between EVAR and open repair of AAA. Patients unfit for open repair should be expected to have more complications, and are at higher risk for endovascular repair than fit patients. Ruptured AAA can be approached via the endovascular technique, and has been shown to have improved long-term survival in certain studies.

\section{Disclosure}

The authors declare no conflicts of interest in this work.

\section{References}

1. Parodi JC, Palmaz JC, Barone HD. Transfemoral intraluminal graft implantation for abdominal aortic aneurysms. Ann Vasc Surg. 1991;5(6): 491-499.

2. Zarins CK, White RA, Schwarten D, et al. AneuRx stent graft versus open surgical repair of abdominal aortic aneurysms: multicenter prospective clinical trial. J Vasc Surg. 1999;29(2):292-305.

3. Powell JT, Brown LC. The natural history of abdominal aortic aneurysms and their risk of rupture. Acta Chir Belg. 2001;101(1):11-16.

4. Lederle FA, Wilson SE, Johnson GR, et al. Immediate repair compared with surveillance of small abdominal aortic aneurysms. N Engl J Med. 2002;346(19):1437-1444.

5. [No authors listed]. Mortality results for randomized controlled trial of early elective surgery or ultrasonographic surveillance for small abdominal aortic aneurysms. The UK Small Aneurysm Trial participants. Lancet. 1998;352(9141):1649-1655.

6. Ouriel K, Clair DG, Kent KC, Zarins CK; Positive Impact of Endovascular Options for Treating Aneurysms Early (PIVOTAL) investigators. Endovascular repair compared with surveillance for patients with small abdominal aortic aneurysms. J Vasc Surg. 2010;51(5):1081-1087.

7. Cao P, De Rango P, Verzini F, et al. Comparison of surveillance versus aortic endografting for small aneurysm repair (CAESAR): results from a randomised trial. Eur J Vasc Endovasc Surg. 2011;41(1):13-25.

8. De Rango P, Verzini F, Parlani G, et al. Quality of life in patients with small abdominal aortic aneurysm: the effect of early endovascular repair versus surveillance in the CAESAR trial. Eur J Vasc Endovasc Surg. 2011;41(3):324-331.

9. Filardo G, Powell JT, Martinez MA, Ballard DJ. Surgery for small asymptomatic abdominal aortic aneurysms. Cochrane Database Syst Rev. 2012;3:CD001835.

10. Leurs LJ, Buth J, Laheij RJ. Long-term results of endovascular abdominal aortic aneurysm treatment with the first generation of commercially available stent grafts. Arch Surg. 2007;142(1):33-41.

11. Abbruzzese TA, Kwolek CJ, Brewster DC, et al. Outcomes following endovascular abdominal aortic aneurysm repair (EVAR): an anatomic and device-specific analysis. J Vasc Surg. 2008;48(1):19-28. 
12. Prinssen M, Verhoeven EL, Buth J, et al. A randomized trial comparing conventional and endovascular repair of abdominal aortic aneurysms. N Engl J Med. 2004;351(16):1607-1618.

13. Blankensteijn JD, de Jong SE, Prinssen M, et al. Two-year outcomes after conventional or endovascular repair of abdominal aortic aneurysms. N Engl J Med. 2005;352(23):2398-2405.

14. De Bruin JL, Baas AF, Buth J, et al. Long-term outcome of open or endovascular repair of abdominal aortic aneurysm. $N$ Engl J Med. 2010;362(20):1881-1889.

15. Greenhalgh RM, Brown LC, Kwong GP, Powell JT, Thompson SG; EVAR trial participants. Comparison of endovascular aneurysm repair with open repair in patients with abdominal aortic aneurysm (EVAR trial 1), 30-day operative mortality results: randomised controlled trial. Lancet. 2004;364(9437):843-848.

16. United Kingdom EVAR Trial investigators, Greenhalgh RM, Brown LC, et al. Endovascular versus open repair of abdominal aortic aneurysm. N Engl J Med. 2010;362(20):1863-1871.

17. Lederle FA, Freischlag JA, Kyriakides TC, et al. Outcomes following endovascular vs open repair of abdominal aortic aneurysm: a randomized trial. JAMA. 2009;302(14):1535-1542.

18. Lederle FA, Freischlag JA, Kyriakides TC, et al. Long-term comparison of endovascular and open repair of abdominal aortic aneurysm. $N$ Engl J Med. 2012;367(21):1988-1997.

19. Prinssen M, Buskens E, Blankensteijn JD; DREAM trial participants. Quality of life endovascular and open AAA repair. Results of a randomised trial. Eur J Vasc Endovasc Surg. 2004;27(2):121-127.

20. Prinssen M, Buskens E, de Jong SE, et al. Cost-effectiveness of conventional and endovascular repair of abdominal aortic aneurysms: results of a randomized trial. J Vasc Surg. 2007;46(5):883-890.

21. Stroupe KT, Lederle FA, Matsumura JS, et al. Cost-effectiveness of open versus endovascular repair of abdominal aortic aneurysm in the OVER trial. J Vasc Surg. 2012;56(4):901-909.

22. United Kingdom EVAR Trial investigators, Greenhalgh RM, Brown LC, Powell JT, Thompson SG, Epstein D. Endovascular repair of aortic aneurysm in patients physically ineligible for open repair. $N E n g l$ J Med. 2010;362(20):1872-1880.
23. Pol RA, Zeebregts CJ, van Sterkenburg SM, Reijnen MM; ENGAGE investigators. Thirty-day outcome and quality of life after endovascular abdominal aortic aneurysm repair in octogenarians based on the Endurant Stent Graft Natural Selection Global Postmarket Registry (ENGAGE). J Vasc Surg. 2012;56(1):27-35.

24. Mehta M, Byrne J, Darling RC 3rd, et al. Endovascular repair of ruptured infrarenal abdominal aortic aneurysm is associated with lower 30-day mortality and better 5-year survival rates than open surgical repair J Vasc Surg. 2013;57(2):368-375.

25. Mayer D, Aeschbacher S, Pfammatter T, et al. Complete replacement of open repair for ruptured abdominal aortic aneurysms by endovascular aneurysm repair: a two-center 14-year experience. Ann Surg. 2012; 256(5):688-695.

26. Takagi H, Umemoto T. A meta-analysis of randomized and risk-adjusted observational studies of endovascular versus open repair for ruptured abdominal aortic aneurysm. Vasc Endovascular Surg. 2011;45(8):717-719.

27. Hinchliffe RJ, Bruijstens L, MacSweeney ST, Braithwaite BD. A randomised trial of endovascular and open surgery for ruptured abdominal aortic aneurysm - results of a pilot study and lessons learned for future studies. Eur J Vasc Endovasc Surg. 2006;32(5):506-513.

28. Hoornweg LL, Wisselink W, Vahl A, Balm R; Amsterdam Acute Aneurysm Trial Collaborators. The Amsterdam Acute Aneurysm Trial: suitability and application rate for endovascular repair of ruptured abdominal aortic aneurysms. Eur J Vasc Endovasc Surg. 2007;33(6):679-683.

29. IMPROVE trial, Powell JT, Thompson SG, et al. The Immediate Management of the Patient with Rupture: Open Versus Endovascular repair (IMPROVE) aneurysm trial - ISRCTN 48334791 IMPROVE trialists. Acta Chir Belg. 2009;109(6):678-680.
Vascular Health and Risk Management

\section{Publish your work in this journal}

Vascular Health and Risk Management is an international, peerreviewed journal of therapeutics and risk management, focusing on concise rapid reporting of clinical studies on the processes involved in the maintenance of vascular health; the monitoring, prevention and treatment of vascular disease and its sequelae; and the involvement of

\section{Dovepress}

metabolic disorders, particularly diabetes. This journal is indexed on PubMed Central and MedLine. The manuscript management system is completely online and includes a very quick and fair peer-review system, which is all easy to use. Visit http://www.dovepress.com/ testimonials.php to read real quotes from published authors. 